\title{
SOLAR EQUATORIAL ROTATION RATE INFERRED FROM INVERSION OF FREQUENCY SPLITTING OF HIGH-DEGREE MODES
}

\author{
Frank Hill \\ National Solar Observatory, National Optical Astronomy Observatories, \\ Tucson, AZ 85726-6732, USA \\ Douglas Gough \\ Institute of Astronomy, and Department of Applied Mathematics and \\ Theoretical Physics, University of Cambridge, Cambridge CB3 OHA, England \\ Juri Toomre and Deborah A. Haber \\ Joint Institute for Laboratory Astrophysics, and Department of Astrophysical, \\ Planetary and Atmospheric Sciences, University of Colorado, \\ Boulder, CO 80309-0440, USA
}

\begin{abstract}
The equatorial rotation rate has been inferred as a function of depth through the outer $16 \mathrm{Mm}$ of the Sun from observations of high-degree five-minute oscillations. An optimal averaging inversion procedure due to Backus \& Gilbert (1970) has been applied to frequency splittings measured from power spectra obtained using Doppler data spanning three and five consecutive days. The resulting rotation curves have proven to be much more stable than the curves obtained from data sets of single days. The results imply that the solar rotation rate increases with depth by $0.023 \mu \mathrm{Hz}$ reaching a maximum at about $2 \mathrm{Mm}$ below the surface, then decreases by $0.037 \mu \mathrm{Hz}$ down to $16 \mathrm{Mm}$.
\end{abstract}

\section{INTRODUCTION}

Helioseismic data have provided us with estimates of the equatorial rotation rate as a function of depth for most of the solar interior (e.g. Duvall et al. 1984, Brown 1985, Libbrecht 1986). These full disk studies have so far been concerned with modes of degree $\ell<100$. It is partly because these modes sample relatively deep portions of the solar interior, and because the frequency range attainable is quite narrow, that the rotation rate in the outermost layers of the convection zone has not been accessible to these studies.

Deubner, Ulrich \& Rhodes (1979) tried to estimate the rotation rate in the outer 15 $\mathrm{Mm}$ of the solar convection zone from high-degree oscillation data. We also have estimated the rotation rate in this region (Hill, Gough \& Toomre 1984), examining separately the power spectra of each individual day of observations. In this study, we reanalyze the data used previously, but we now treat the data in sets spanning several consecutive days. This improves the frequency resolution, reducing the effects of mode beating and stabilizing the resulting rotation curves.

\section{OBSERVATIONS AND ANALYSIS}

The data have been described in some detail by Hill, Gough \& Toomre (1984). Briefly, they consist of Doppler observations made in 1981 with the echelle spectrograph and diode array at the vacuum tower telescope of NSO/Sacramento Peak. The observations were made simultaneously in the red and blue sides of the cores of the two spectral lines Fe I $\lambda 5434$ and $\mathrm{Mg}$ I $\lambda 5173$. The observing window was a narrow rectangle of dimensions 256" 
$\times 1024^{n}$, with the long dimension oriented parallel to the solar equator, and with a nominal $2^{n}$ spatial resolution. The Doppler velocities were averaged along the shorter dimension of the window, effectively filtering out non-sectoral modes. The data were then corrected for foreshortening and Fourier transformed in space and time to produce $m-\nu$ power diagrams, where $m$ is the azimuthal order of a mode and $\nu$ is temporal cyclic frequency.

The major difference between this and our former analysis is the treatment of the data in the temporal domain. Previously we had analyzed each day individually, resulting in a frequency resolution $\Delta \nu$ of $23.89 \mu \mathrm{Hz}$. Here we concatenate consecutive days of the data, forming two independent sets spanning 58.14 hours ( 3 days: 1981; days 53-55) and 104.65 hours (5 days: 1981 ; days 319-323). The frequency resolutions of the reanalyzed data are thus $4.78 \mu \mathrm{Hz}$ for the 3-day set, and $2.65 \mu \mathrm{Hz}$ for the 5-day set. We have analyzed both the $\mathrm{Fe} I$ and the $\mathrm{Mg}$ I data sets which sample the oscillations at different heights in the solar atmosphere.

The frequencies of the oscillations were determined by fitting a variable-knot cubic spline to the ridge centroids. Frequency splittings between prograde and retrograde modes were then computed. The Backus-Gilbert optimal averaging inversion procedure (e.g. Backus \& Gilbert 1970, Gough 1985) was then applied using the frequency splittings of 709 sectoral modes with degree $80 \leq \ell \leq 995$ and order $0 \leq n \leq 8$. A total of four rotation curves were computed, one each for the 3-day and 5-day sets from both the Fe I and the Mg I data.

\section{RESULTS AND DISCUSSION}

The resulting rotation curves for the four data sets are shown in Figure 1. These curves show the sidereal equatorial rotation rate in $\mu \mathrm{Hz}$ as a function of depth. The depth scale is shown in units of the logarithm of the pressure as well as in $\mathrm{Mm}$. The curves are all similar in shape, in contrast to the results we obtained previously when considering the single days separately. Those previous rotation curves displayed large variations that we first ascribed to the passage of giant convective cells beneath the observing window. Numerical experiments have later suggested that the daily variations may be due instead to the beating of unresolved modes. The concatenation of the data reduces mode beating by increasing the frequency resolution of the spectra. The outcome has been to stabilize the shape of the rotation curves.

Figure 2 displays the average of the four rotation curves shown in Figure 1. This curve represents our best determination of the solar sidereal equatorial rotation rate in the outer layers of the convection zone. The curve indicates that the rotation rate increases by about $0.023 \mu \mathrm{Hz}$, or about $100 \mathrm{~ms}^{-1}$, reaching a local maximum at a depth of about 2 $\mathrm{Mm}$. It then decreases by about $0.037 \mu \mathrm{Hz}\left(\sim 150 \mathrm{~ms}^{-1}\right)$ over the depth range 2 to 16 $\mathrm{Mm}$. The behavior of this curve is consistent with the full-disk results that suggest that the rotation rate over much of the solar interior is somewhat lower than the surface rate. It is also perhaps consistent with sunspot tracer measurements of the solar rotation rate which typically show a higher value than that measured from Doppler shifts of the plasma.

We should note that our measurement of the rotation rate near the surface is rather different than the surface rate measured by spectroscopic means. We find a rate of 0.428 $\mu \mathrm{Hz}$ at a depth of $0.2 \mathrm{Mm}$, while the latest Mt. Wilson Doppler rate is $0.454 \mu \mathrm{Hz}$ (Howard 1984). While the presence of a strong shear layer in the outer $0.2 \mathrm{Mm}$ cannot be ruled out by our data, the existence of such a layer would probably result in the creation of other observable instabilities. It is most likely that the zero point of our rotation rate scale is inaccurate, and that the source of the discrepancy is a slow drift of the telescope during the course of the observations. A systematic drift of less than 0.5 " per hour would 


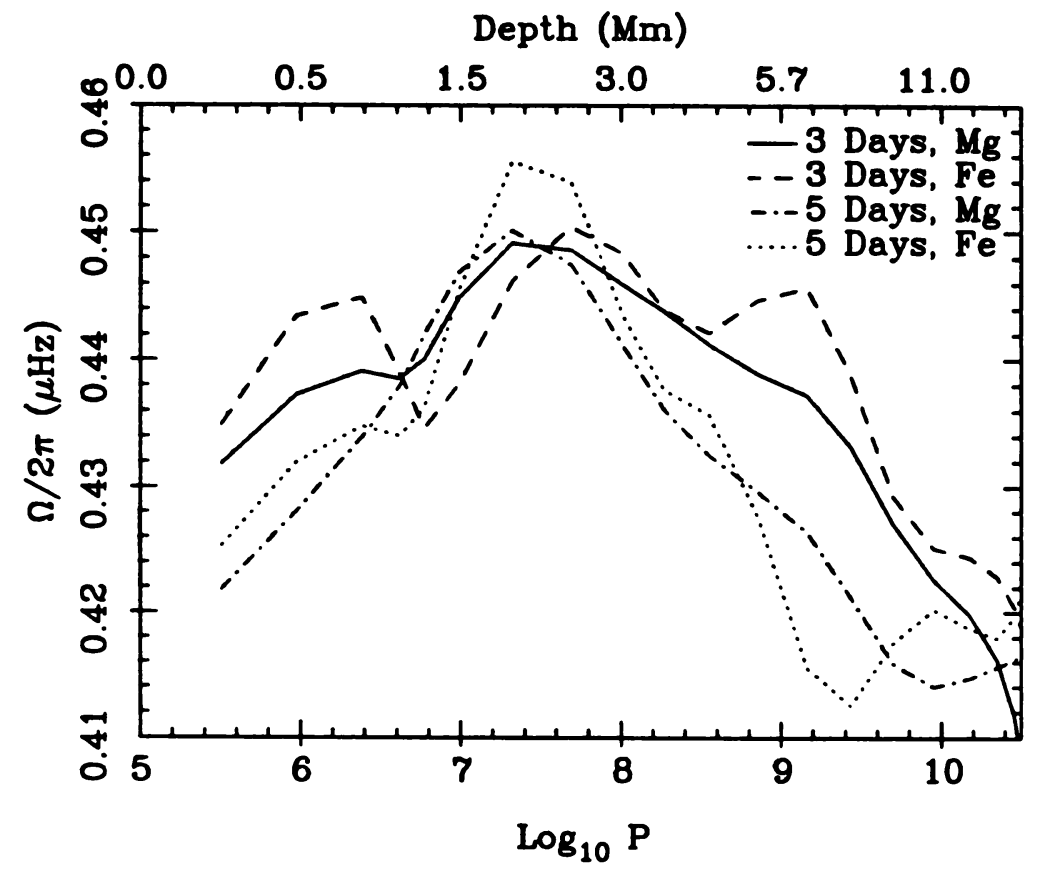

Figure 1. The sidereal equatorial rotation rate as a function of depth inferred from a Backus-Gilbert inversion of the frequency splittings of 709 modes. The four curves show the results for the 3 and 5-day sets obtained in $\mathrm{Fe} \mathrm{I}$ and $\mathrm{Mg} \mathrm{I}$.

be sufficient to explain the discrepancy. Although the telescope was locked to the solar image with a laser during these observations, a residual drift of this magnitude may still have been present. If we allow that there is a zero point offset of about $0.024 \mu \mathrm{Hz}$ in the rotation curve of this study, then the results are consistent with the results of Hill, Rust \& Appourchaux (these proceedings), which sample the layers of the Sun immediately below the depths reached in this study.

We note that the maximum of the rotation rate occurs at approximately the depth of the hydrogen ionization zone. This region has been postulated as the source of the driving for the granulation. It is tempting to speculate that the coincidence of the maximum in the rotation rate with this region is not accidental. However, the relationship between the shear and the granulation is unclear. The variation of rotation rate with depth must be the result of convection redistributing the angular momentum, yet convection with the largest horizontal scales is likely to play the biggest role. The strong driving of smallscale granulation at a depth of about $2 \mathrm{Mm}$ may serve to deflect the large-scale flows of supergranulation and giant cells and prevent them from extending into the atmosphere with any appreciable amplitude. The peak in rotation rate may then reflect the depth at which such interaction between the smallest and largest scales of convection is occurring. Alternatively, the granulation itself may be partly a product of turbulence caused by shear flow instabilities in the vortex sheet. Whatever the mechanism, it is encouraging that we are now beginning to obtain information on the flows that exist in the outer solar convection zone. This information should prove useful to theories of solar convection and the solar dynamo. 


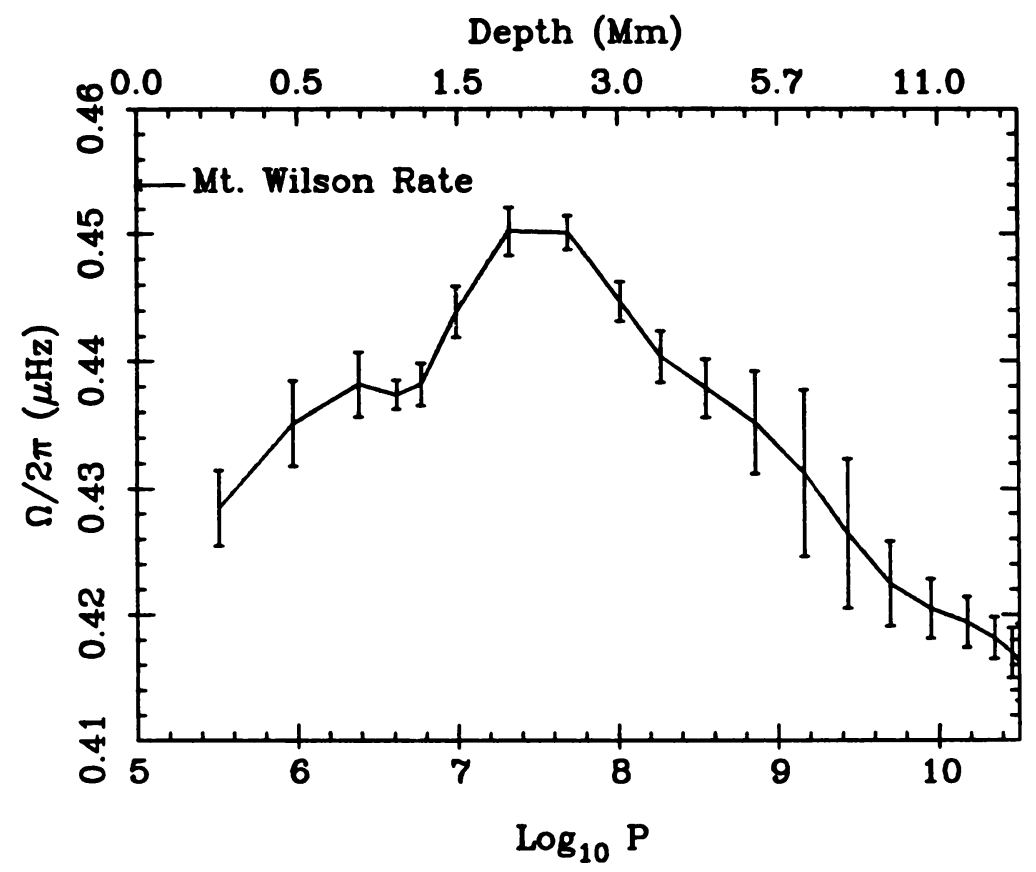

Figure 2. The equatorial rotation rate as a function of depth resulting from averaging the four curves shown in Figure 1. The bars denote the standard deviation from the mean of those four curves.

Acknowledgements - Thanks are due to Larry November, Tom Duvall, Jack Harvey, and John Leibacher for scientific discussions, and to the Sac-Peak Tower support staff for assistance with the observations. The Joint Institute for Laboratory Astrophysics is operated jointly by the National Bureau of Standards and the University of Colorado. The work at Colorado was supported in part by the National Aeronautics and Space Administration through grants NSG-7511, NAGW-91 and NAG-5-513, and by the Air Force Geophysics Laboratory through contract F19628-82-K-0008. The National Optical Astronomy Observatories are operated by the Association of Universities for Research in Astronomy, Inc. under contract with the National Science Foundation.

\section{REFERENCES}

Backus, G.E., and Gilbert, J.F. 1970, Phil. Trans. Roy. Soc. London A 266, 123.

Brown, T.M. 1985, Nature 317, 591.

Deubner, F.-L., Ulrich, R.K., and Rhodes, E.J., Jr. 1979, Astron. \& Astrophys. 72, 177.

Duvall, T.L., Jr., Dziembowski, W., Goode, P.R., Gough, D.O., Harvey, J.W., and Leibacher, J.W. 1984, Nature 310, 22.

Gough, D.O. 1985, Solar Phys, 100, 65.

Hill, F., Gough, D.O., and Toomre, J. 1984, Mem. Soc. Astron. Ital. 55, 153.

Howard, R. 1984, Ann. Rev. Astron. Astrophys. 22, 131.

Libbrecht, K.G. 1986, Nature 319, 753. 